microarchitecture parameters were correlated to each other but not corre lated with BMD. Multiple regression analysis demonstrated that the combination of the microarchitecture parameters and BMD improved the prediction of the failure load with for example an improved fracture risk prediction from $\mathrm{R}^{2}=0.418$ to 0.688 when combining $\mathrm{BMD}$ and Euler $\mathrm{Ch}$ Overall, femur bone microarchitecture assessed with UHF MRI was significantly correlated with biomechanical parameters. The multimodal assess ment of BMD and trabecular bone microarchitecture using UHF MR improved the fracture risk prediction of femoral bone and might be of interest for the future investigation of selected osteoporotic patients.

Conclusion: We demonstrated that femur bone microarchitecture assessed with UHF MRI was significantly correlated with biomechanical parameters. The multimodal assessment of bone mineral density and trabecular bone microarchitecture using UHF MRI improved the fracture risk prediction of femoral bone and might be of interest for the future investigation of selected osteoporotic patients.

Disclosure of Interests: None declared

DOI: 10.1136/annrheumdis-2019-eular.2556

\section{AB1145 1 FULLY CONVOLUTIONAL NEURAL NETWORK-BASED SEGMENTATION OF INDIVIDUAL MUSCLES IN MR IMAGES USING MUSCLES AND BORDERS PARCELLATIONS}

Joris Fournel' ${ }^{1}$, Arnaud Le Troter ${ }^{1}$, Sandrine Guis ${ }^{2}$, David Bendahan ${ }^{1}$, Badih Ghattas ${ }^{3} .{ }^{1}$ Aix-Marseille Univ, CNRS, CRMBM UMR 7339, Marseille, France; ${ }^{2}$ Aix-Marseille Univ, CNRS, AP-HM, CRMBM UMR 7339, Rheumatology, Marseille, France; ${ }^{3}$ Aix-Marseille Univ, CNRS, Institut de Mathématiques de Marseille, UMR 7373, Marseille, France

Background: Segmentation of individual muscles in MR images is challenging considering the poor contrast between muscles and the large variability between and within subjects.

Objectives: The segmentation performance of the Bayesian SegNet network was assessed for the four individual muscles of the quadriceps group. In addition to the classes corresponding to each muscle, we analyzed the effect of adding four additional classes corresponding to muscle borders. We also investigated the network performance taking into account each muscle individually or the whole set of muscles. The corresponding results were compared with those obtained using a conventional multi-atlas method.

Methods: For the training phase, a dataset of 500 images was used while the testing phase was performed for two other datasets with 140 images each. Four different variants of the same network were assayed considering simultaneous segmentation of individual muscles (On5), separate segmentation of individual muscles (Fn2) and the use of additional classes related to muscle borders in both cases (On9 and Fn3).

Results: All approaches largely outperformed the results of a multi-atlas strategy. The higher DSI values i.e. $0.96 \pm 0.01$ for the rectus femoris muscle, $0.93 \pm 0.01$ for the vastus intermedius muscle, $0.94 \pm 0.03$ for the vastus lateralis muscle and $0.96 \pm 0.01$ for the vastus medialis muscle were obtained with the On9 and Fn3 networks i.e. taking into account the muscle borders labels in addition to the muscle labels.

Conclusion: Deep-learning based methods are optimal for the segmentation of thigh muscles and the corresponding efficiency can be improved when considering labels for muscles together with borders.

Disclosure of Interests: None declared

DOI: 10.1136/annrheumdis-2019-eular.2804

\section{AB1146 PREVALENCE OF STRUCTURAL CHANGES AT THE POSTERIOR PART OF THE SPINE IN AXIAL SPONDYLOARTHRITIS EVALUATED WITH MR}

Andreas Haidmayer ${ }^{1}$, Gabriel Adelsmayr ${ }^{2}$, Rusmir Husic ${ }^{1}$, Franz Quehenberger ${ }^{3}$, Angelika Lackner ${ }^{1}$, Joesf Hermann ${ }^{1}$. ${ }^{1}$ Medical University of Graz, Internal Medicine, Division for Rheumatology and Immunology, Graz, Austria; ${ }^{2}$ Medical University of Graz, Radiology, Division for general Radiology, Graz, Austria; ${ }^{3}$ Medical University of Graz, Institute for Medical Information, Statistics and Documentation, Graz, Austria

Background: In clinical trials radiographic damage is frequently quantified by conventional $x$-ray but neither the thoracic spine nor the posterior parts of the vertebral column including the intervertebral joints, the vertebral arch and the spinous process can be evaluated reliably with this instrument. Bone inflammation and areas of enthesitis can easily be detected by magnetic tomography imaging (MRI) and the introduction of high-resolution 3-tesla MRI allows analysis of bone structures more precisely than with $\mathrm{x}$-ray without radiation exposure [1]

Patients with axial $\mathrm{SpA}$ frequently present with reduced spinal mobility and functional impairment, but radiographic signs of ankylosis are missing on plain radiographs. These particular clinical findings are reinforced by data in the literature showing, that the relation between radiographic damage, spinal mobility and function is low. Radiographic damage to the posterior parts of the vertebral column may be responsible for the discrepancy found between clinical and radiographic results as suggested by a Dutch group [2].

Objectives: To evaluate the localisation and incidence of new bone formation in the posterior part of the vertebral bodies with MRI

Methods: In a cohort of 56 patients with diagnosed axial SpA (mean age 50.29y, mean disease duration $14.4 y, 76.4 \%$ men, $88.7 \%$ HLA-B27 positive) we performed a MRI (3 Tesla scanner Skyra, Siemens Healthcare, Erlangen, Germany). The whole spine was scanned with T1 weighted and Turbo-Inversion Recovery Magnitude (TIRM) sequences in sagittal planes and each segment of the vertebral column was divided into four areas (ventral and posterior part of the vertebral bodies, intervertebral joints andspinous processes) to assess osseous changes like sclerosis, erosion, syndesmophytes, ankylosis, (partial) fusion of intervertebral joints and fibroostosis of spinous processes.

Results: Syndesmophytes and ankylosis of the ventral segments were present from C7/Th1 down to L5/S1 with a maximum in Th5/6 (62.5\%). In the posterior segments of the vertebral bodies we detected a contrary accumulation inside the cervical and lumbar spine with a max. in C5/6 $(55.4 \%)$ respectively $\mathrm{L} 2 / 3(66.1 \%)$. Erosions were mainly found at the ventral edges with an accumulation in middle cervical, lower thoracic and the whole lumbar spine (max. Th9/10 24.5\%)

Ankylosis of the intervertebral joints was present over the whole spine with a preference of the cervical (max. C5/6 27.7\%) and lumbar column (max. L2/3 47.3\%). Fibroostosis of the spinous processeswere rarely found in the middle and lower thoracic spine (Th5-11, each 2.7\%).

In up to $57.10 \%$ of the cervical spine $(54.78 \%$ for lumbar, $32.18 \%$ for thoracic spine) with detectable new bone formation of the posterior seg ments we found no changes of the ventral part.

Conclusion: With high resolution MRI we were able to detect structural in the dorsal part of the spine, which can hardly be assessed by conventional radiographs. These findings may explain functional disability in patients with non-corresponding findings in $\mathrm{x}$-ray.

\section{REFERENCES}

[1] Lee $\mathrm{CH}$, et al. (2015) Correlation of structural abnormalities of the wrist and metacarpophalangeal joints evaluated by high-resolution peripheral quantitative computed tomography, 3 Tesla magnetic resonance imaging and conventional radiographs in rheumatoid arthritis. International journal of rheumatic diseases

[2] Maas F, et al. (2015) Comparison of radiographic damage of the zygapophyseal joints and the vertebral bodies of the cervical spine in patients with ankylosing spondylitis before and after 4 years of TNF-alpha blocking therapy. Annals of the rheumatic diseases

Disclosure of Interests: Andreas Haidmayer Speakers bureau: Roche, MSD, BMS, Abbvie, Celgene, Gabriel Adelsmayr Speakers bureau: BMS, Rusmir Husic Speakers bureau: BMS, UCB, Celgene, MSD, Franz Quehenberger: None declared, Angelika Lackner: None declared, Joesf Hermann Speakers bureau: Abbvie, MSD, UCB, BMS, Celgene

DOI: 10.1136/annrheumdis-2019-eular.7267

\section{$\mathrm{AB} 1147$ VERTEBRAL FRACTURE ASSESSMENT : A SIMPLE TOOL TO DETECT VERTEBRAL FRACTURE IN THE OSTEOPOROSIS ASSESSMENT OF PATIENTS WITH TYPE2 DIABETES MELLITUS}

Nassima Hanouchine ${ }^{1}$, Salah Ouhadj ${ }^{2}$, Chafia Dahou-Makhloufi ${ }^{3} .{ }^{1}$ chu tizi ouzou, TIZI OUZOU, Algeria; ${ }^{2}$ EPH DELLYS, BOUMERDES, Algeria; ${ }^{3} \mathrm{CHU} B A B$ ELOUED, ALGIERS, Algeria

Background: Vertebral fracture assessment ( VFA) has several benefits including substantially lower radiation dose, lower cost, higher patient convenience, and less operator-dependent variance,VFA can be directly assessed during bone mineral density ( BMD) measurement

Objectives: evaluate the accuracy of VFA performed in the supine position by using conventional visual radiography of the spine as the reference standard in patients with type 2 diabetes mellitus

Methods: A total of 207 patients with type 2 diabetes mellitus (mean age, 46,59 $\pm 7,53$ years; range, 21-60 years) consisting of 102 women 
and 105 men,who were suspected of having osteoporosis and who underwent VFA in the supine position and radiography of the spine were evaluated. VFA was analyzed by using a six-marker point method to describe the shape and deformity of each vertebra. Visual radiography of the lateral spine was performed by an experienced radiologist. The agreement between VFA and visual radiography, was assessed by using weighted statistics.

Results: Visual radiography helped identify 51 (24.6\%) patients with at least one vertebral fracture versus $49(23,67 \%)$ with VFA. Most fractures were present in T7, T12, and L1. Excellent agreement was found between VFA and visual radiography, with $97.3 \%$ concordance and 0.89 . Sensitivity, specificity, and positive and negative predictive values calculated by lesion level for VFA compared with visual assessment were $90.2 \%, 98.08 \%, 93.88 \%$, and $96.84 \%$, respectively.

Conclusion: VFA performed with patients with type 2 diabetes, in the supine position, is an accurate method to help detect vertebral fractures when compared with conventional spine radiography. VFA permits combination of fracture assessment with bone mineral density measurement in a single session

\section{REFERENCES}

[1] -Jacobs-Kosmin D, Sandorfi N, Murray H, Abruzzo JL. Vertebral deformities identified by vertebral fracture assessment: associations with clinical characteristics and bone mineral density. J Clin Densitom 2005;8: 267272.

[2] -Binkley N, Krueger D, Gangnon R, Genant HK, Drezner MK. Lateral vertebral assessment: a valuable technique to detect clinically significant vertebral fractures. Osteoporos Int 2005;16:1513-1518.

[3] -Schousboe JT, DeBold CR. Reliability and accuracy of vertebral fracture assessment with densitometry compared to radiography in clinical practice. Osteoporos Int 2006;17: 281-289

Disclosure of Interests: None declared

DOI: 10.1136/annrheumdis-2019-eular.8012

\section{AB1148 THE RELATION ANALYSIS OF BONE MICROARCHITECTURE EVALUATED BY HR-PQCT, AND SYNOVITIS, BONE DESTRUCTION, SYSTEMIC OSTEOPOROSIS IN RHEUMATOID ARTHRITIS}

Naoki Iwamoto ${ }^{1}$, Konosuke Watanabe ${ }^{2}$, Tomohiro Koga ${ }^{1}$, Shin-Ya Kawashiri ${ }^{1}$, Kunihiro Ichinose ${ }^{1}$, Mami Tamai ${ }^{1}$, Hideki Nakamura ${ }^{1}$, Nozomi Ohki ${ }^{3}$, Ko Chiba ${ }^{2}$, Tomoki Origuchi ${ }^{4}$, Makoto Osaki ${ }^{2}$, Atsushi Kawakami ${ }^{1}{ }^{1}$ Nagasaki University Graduate School of Biomedical Sciences, Department of Immunology and Rheumatology, Division of Advanced Preventive Medical Sciences, Nagasaki, Japan; ${ }^{2}$ Nagasaki University Graduate School of Biomedical Sciences, Department of Orthopedic Surgery, Nagasaki, Japan; ${ }^{3}$ Nagasaki University Graduate School of Biomedical Sciences, Department of Radiological Sciences, Nagasaki, Japan; ${ }^{4}$ Nagasaki University Graduate School of Biomedical Sciences, Department of Physical Therapy, Nagasaki, Japan

Background: Periarticular osteoporosis is one of hallmark of rheumatoid arthritis (RA). However, until now the periarticular bone structure including bone mineral density have not been fully elucidated. High-resolution peripheral quantitative computed tomography (HR-pQCT) is a new technique with high spatial resolution that enables us to assess microarchitecture of cancellous and cortical bones that cannot be assessed by conventional X-ray examinations. Recently, few studies using HR-pQCT revealed that bone microarchitecture such as trabecular volumetric densities (Tb.vBMD) were different between RA and non-RA ${ }^{1}$, but these study had not compared findings of HR-pQCT with synovitis assessed by ultrasonography(US) or systemic osteoporosis.

Objectives: To investigate bone microarchitecture evaluated by HR-pQCT in RA.

Methods: This study included 21 RA patient. HR-pQCT imaging analyses quantified bone microarchitecture in 2,3 Metacarpal Head. We measured the bone mineral density (BMD) of lumber spine and femoral neck using Dual-Energy X-ray Absorptiometry (DXA). Synovitis and bone destruction were assessed by US and X-ray, respectively.

Results: Disease duration, age and disease activity were not correlated with bone microarchitecture. BMD of femoral neck was correlated with Tb.vBMD $(r=0.84, p<0.01)$. The joints with US-proven active synovitis [power doppler score $(P D) \geq 2$ )] showed less Tb.vBMD, trabecular number (Tb.N) and trabecular thickness (Tb.Th) as compared with the patients with US-PD<2 synovitis (Tb.vBMD:121.5 mg/cm ${ }^{3}$ vs $145.3 \mathrm{mg} / \mathrm{cm}^{3}$, Figure 1). These tendencies were also shown in deferent Metacarpal Heads in the same patient (the mean difference of Tb.vBMD, $P D \geq 2-P D<2$ : -11.9 $\mathrm{mg} / \mathrm{cm}^{3}$ ). Moreover, the joints with progressive joint destruction as classified by more than steinbrocker stage 3 showed less Tb.vBMD (122.1 $\mathrm{mg} / \mathrm{cm}^{3}$ vs $150.0 \mathrm{mg} / \mathrm{cm}^{3}$ ). The longitudinal analysis of 10 patients revealed that Tb.vBMD and TB.N were improved along with improvement of disease activity (DAS -2.80: from baseline to 12 months after new treatment initiated, but $\mathrm{Tb}$.Th was not improved.

Conclusion: This study revealed that bone destruction and synovitis were associated with bone microarchitecture and, the difference of treatment response by parameter of bone microarchitecture. However, this study was mainly transverse analysis and small samples, we need longitudinal analysis using larger samples.

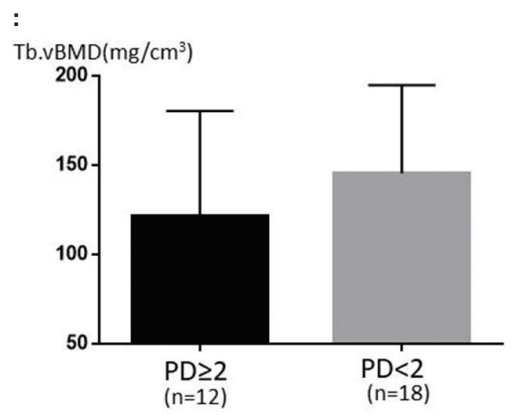

Abstract AB1148 Figure 1

\section{REFERENCES}

[1] Feehan LM, Li LL, McKay HA. Micro-structural bone changes in early rheumatoid arthritis persist over 1-year despite use of disease modifying anti-rheumatic drug therapy. BMC Musculoskelet Disord 2017; 18(1): 521.

Disclosure of Interests: Naoki Iwamoto: None declared, Konosuke Watanabe: None declared, Tomohiro Koga: None declared, Shin-ya Kawashiri: None declared, Kunihiro Ichinose: None declared, Mami Tamai: None declared, Hideki Nakamura: None declared, Nozomi Ohki: None declared, Ko Chiba: None declared, Tomoki Origuchi: None declared, Makoto Osaki: None declared, Atsushi Kawakami Grant/research support from: Astellas Pharma, Consultant for: Astellas Pharma, Speakers bureau: Astellas Pharma

DOI: 10.1136/annrheumdis-2019-eular.5484

\section{AB1149 CHARACTERIZATION OF SALIVARY PROTEINS IDENTIFIED AS POTENTIAL BIOMARKERS FOR SYSTEMIC LUPUS ERYTHEMATOSUS THROUGH PROTEOMIC ANALYSIS}

Ju-Yang Jung, Jiwon Kim, Hyoun-Ah Kim, Chang-Hee Suh. Ajou University School of Medicine, Suwon, Korea, Rep. of (South Korea)

Background: Systemic lupus erythematosus (SLE) is a heterogeneous autoimmune disease characterized by pathogenic autoantibodies and uncontrolled inflammatory response. There are few reliable biomarkers available for diagnosis and monitoring the disease.

Objectives: We tried to find and characterize specific protein components in saliva of patients with SLE for their use as biomarkers in future. Methods: Salivary proteins were prepared from 11 samples from patients with SLE and healthy controls $(\mathrm{HC})$, and were subjected to 2-dimensional gel electrophoresis (2-DE). The spots with greater than 2 fold change in intensity were identified by matrix-assisted laser desorption/ionization timeof-flight mass spectrometer (MS) analysis. The relative and absolute amounts of the several candidate proteins in saliva of patients with SLE and rheumatoid arthritis (RA), and $\mathrm{HC}$ were analyzed using western blotting, and enzyme-linked immunosorbent assay.

Results: Proteomic analysis using 2-DE and MS identified 20 differentially expressed protein spots in the saliva of SLE patients compared in that of $\mathrm{HC}$. Among them, proteins with more than two-fold differences in expression were found as immunoglobulin gamma-3 chain C (IGHG3), immunoglobulin alpha-1 chain C region, protein S100, lactotransferrin, leukemia-associated protein 7, and 8-oxoguanine DNA glycosylase (OGG1). salivary IGHG3 levels were increased in SLE (3.9 $\pm 2.2 \mathrm{pg} / \mathrm{mL}) \mathrm{com}$ pared to those in RA $(1.8 \pm 1.0 \mathrm{pg} / \mathrm{mL}, \mathrm{p}<0.001)$ or $\mathrm{HC}(2.1 \pm 1.6$ $\mathrm{pg} / \mathrm{mL}, \mathrm{p}<0.001)$, and salivary lactotransferrin levels were increased in SLE $(5.0 \pm 1.7 \mathrm{pg} / \mathrm{mL})$ compared to those in RA $(3.1 \pm 1.6 \mathrm{pg} / \mathrm{mL}, \mathrm{p}<$ $0.001)$ or $\mathrm{HC}(2.3 \pm 1.7 \mathrm{pg} / \mathrm{mL}, \mathrm{p}<0.001)$. The patients with nephritis had higher salivary IGHG3 $(4.7 \pm 1.9 \mathrm{pg} / \mathrm{mL})$ than those not $(3.6 \pm 2.2$ 